\title{
Evaluation of Structural Degradation of Polymeric Composites Subjected to Self-Heating by the Thermal Diffusivity Analysis
}

\author{
Andrzej Katunin ${ }^{1}$ - Sebastian Pawlak ${ }^{2}$ - Angelika Wronkowicz ${ }^{1}$
}

Received: 13 April 2017 / Accepted: 22 October 2017 / Published online: 13 November 2017

(C) The Author(s) 2017. This article is an open access publication

\begin{abstract}
A significant acceleration of degradation process is observed during occurrence of the self-heating effect, which has a character of mechanical energy dissipation and causes heating-up of structures. Following this, it is essential to evaluate a critical value of self-heating temperature at which a thermal domination of fatigue processes occurs. With a progress of degradation irreversible structural changes occur. In this study, an experimental evaluation of the degradation process was performed based on analysis of apparent thermal diffusivity of a composite structure at various selfheating temperature values. The obtained results show that the estimated apparent thermal diffusivity is a sensitive measure which allows for effective evaluation of structural changes caused by fatigue processes.
\end{abstract}

Keywords Self-heating effect - Apparent thermal diffusivity $\cdot$ Composite structures $\cdot$ Damage initiation

\section{Introduction}

Since polymeric composites have found wide application in many structural elements subjected to vibrations or cyclic loading, at certain loading conditions a self-heating effect may appear. This effect reflects in heating-up of a loaded structure due to dissipation of energy resulting from out-

Andrzej Katunin

andrzej.katunin@polsl.pl

1 Institute of Fundamentals of Machinery Design, Faculty of Mechanical Engineering, Silesian University of Technology, Konarskiego 18A, 44-100 Gliwice, Poland

2 Department of Welding, Faculty of Mechanical Engineering, Silesian University of Technology, Konarskiego 18A, 44-100 Gliwice, Poland of-phase oscillations between stress and strain magnitudes and, depending on character of its development, may significantly intensify fatigue processes and result in quick degradation and rupture of the structure [1]. This is because a major amount of dissipated mechanical energy transforms into heat; however, a small amount of this energy dissipates toward development of plastic mechanisms [2] and microcracking [3]. The degradation process of a composite structure under conditions of fatigue loading accompanied with the self-heating effect can be featured with a characteristic three-stage degradation model, which is observable both in decrease in mechanical properties $[4,5]$ as well as in evolution of the self-heating temperature $[1,6,7]$. The selfheating effect, due to the character of its development, can be classified into two types: In the first case, when stationary self-heating occurs, the self-heating temperature grows until reaching a certain temperature value and stabilizes at this value due to reaching a thermal equilibrium between the generated and convected thermal energy, while in the second case, the self-heating temperature growth has nonstationary character, which leads to significant influence and domination on the fatigue process. The stabilization of the self-heating temperature in the stationary self-heating process usually lasts for a long period of time, and, besides the influence on the fatigue process, it does not dominate it. In this case, the first and the second phases of self-heating are observable. In contrast to this case, the non-stationary selfheating process usually dominates fatigue processes, which results in a significant intensification of degradation.

In order to investigate fracture mechanisms occurring during the degradation process of a polymeric composite structure subjected to self-heating an evolution of material properties of the structure and operational parameters of loading can be analyzed. The previous studies $[8,9]$ include an analysis of evolution character of loading force, deflec- 
tion velocity and self-heating surface temperature, and the performed analyses have shown low effectiveness in degradation degree evaluation of the two first mentioned parameters. The changes visible in these characteristics were observable only when a resulting damage was well developed and visible by a naked eye on the tested structure. The selfheating temperature evolution gave much better results in detection of fatigue damage resulted from a loading process. The technique proposed in [9], based on approximation of a self-heating temperature history curve, gives information about the beginning of the third phase of structural degradation, namely the non-stationary macrocrack development. Another valuable approach of identification of damage initiation is an evaluation of the degradation degree of composite structures through measurements of acoustic emission (AE), being intensively developed by Kahirdeh and Khonsari [10]. This method is sufficiently sensitive to detect initial cracking occurring in a matrix of a composite during cyclic loading, and thus, it can be considered as an effective parameter for the degradation degree evaluation in the fatigue process. Recent authors' studies have shown that sensitivity of the temperature- and AE-based techniques is comparable [11].

Although the temperature- and AE-based techniques are more effective than the previously described ones, all of the considered parameters reveal to be indirect measures of the structural degradation. Therefore, for damage detection in a possibly early stage of its development an analysis of more sensitive physical properties is necessary. An increase in the sensitivity is possible to be achieved by considering material properties, since due to the development of damage at first the material properties will locally change. (The indirect parameters, like the loading force or surface temperature, change as a consequence of changing the material properties.) As a good indicator of structural changes resulting from selfheating one can consider elastic modulus or a critical yield stress of a material; however, in order to perform such tests the specimens must be destroyed.

One of such material properties is a thermal diffusivity (TD) of a tested structure. From a variety of thermal properties of materials, TD seems to be the most relevant, since it contains such properties as a thermal conductivity and a heat capacity, which also change during structural degradation. Moreover, TD can be used as an indicator of how quickly and thoroughly a temperature value of a material changes in response to heat excitation [12]. Observed changes of TD can be considered, in turn, as a direct indicator of structural degradation. Experimental measurements of TD can be easily performed using a classical approach based on a flash diffusivity method proposed by Parker et al. [13]. Firstly, this method found application in determination of TD in homogeneous materials, including polymers [13-15]; however, further, it was also extended to determination of TD in heterogeneous materials, like polymeric composites $[13,16]$.
Considering a fact that TD in the investigated problem is determined over an area with damage, one should note that determined value of TD is, in fact, a combination of TD of a composite specimen and TD of air gaps resulting from fracture. Therefore, for clarity of naming convention, the measured parameter during the tests in damaged region will be further called the apparent thermal diffusivity (ATD).

The presented study focuses on evaluation of influence of the degradation degree of a composite structure subjected to fatigue with the accompanying self-heating effect on changes of its thermal properties, in particular, ATD. The main goal of this study is to determine a functional dependency between the degradation degree and ATD and, based on the obtained results, reevaluate a criticality of the self-heating effect in terms of its domination on the fatigue process of polymeric composites. In order to verify effectiveness of the proposed approach of degradation degree evaluation based on ATD, the obtained results were compared with result obtained from self-heating temperature and AE measurements. Knowledge of criticality of the self-heating effect can be very helpful both during the design and operation of elements made of polymeric composites as well as during prognosing of their residual life.

\section{Fatigue Testing}

Glass fabric-reinforced epoxy composite specimens for fatigue tests were manufactured and supplied by Izo-Erg S.A. (Gliwice, Poland). The composite contains 14 unidirectional layers with a fiber content of $30 \%$ of its whole weight. A description of manufacturing procedure and mechanical properties of the composite can be found in [17]. Due to a relatively high content of the polymer in the resulting composite, the self-heating effect develops well during the cyclic loading. Specific dimensions of the specimens were as follows: the length of $100 \mathrm{~mm}$, the width of $10 \mathrm{~mm}$ and the thickness of $2.5 \mathrm{~mm}$. In order to investigate the degradation degree, the specimens were cyclically loaded using an experimental test rig until reaching a defined self-heating temperature. The test rig is presented in Fig. 1. A specimen 5 was loaded using electrodynamic shaker TIRA@ $($ TV-51120 1 controlled by a PC 14 with the own-developed application. The application allows controlling the excitation signal parameters through an analogue output of multi-channel signal acquisition module 11 and controls the shaker amplifier TIRA® BAA 500 10. During loading the following physical parameters were measured: excitation force (using a force sensor PCB Piezotronics® 208C03 7 mounted at the end of an excitation stinger 3 close to an upper holder 6 of the specimen 5), vibration velocity (using laser Doppler vibrometer Polytec $($ PDV-100 9 focused on a point near a lower clamp 4 of the specimen 5), acceleration of an exci- 




(a)

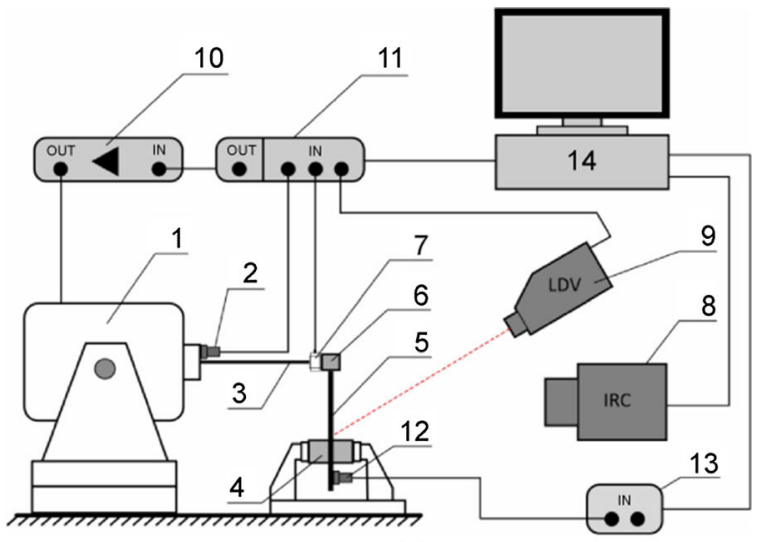

(b)

Fig. 1 A picture (a) and a scheme (b) of the experimental test rig for fatigue tests and evaluation of the self-heating effect

tation (using accelerometer PCB Piezotronics(B) T352C34 2 mounted directly on the shaker 1 ), a surface temperature (using an infrared camera Infratec $\mathbb{R}$ ) VarioCAM(R) $\mathrm{hr}$ 8 ) and acoustic emission (using an acoustic emission sensor 12 glued directly to the unloaded part of the tested specimen 5, which, together with an AE signal preamplifier and a dualchannel AE signal processor board 13, constitutes a system Vallen(R) AMSY-5).

Based on the previous experiments and results [9], the self-heating temperature range, reached for specimens used in this study, was assumed as $40-100^{\circ} \mathrm{C}$ with a step of $5^{\circ} \mathrm{C}$, and additional single specimen loaded up to total failure caused by self-heating with a maximal self-heating temperature value of $110^{\circ} \mathrm{C}$. In order to ensure statistically valid measurement data, for each unique set of loading three specimens were tested. This means that all specimens were heated up until reaching the specific self-heating temperature, which resulted in 40 tested specimens (including the totally failed specimen). The initial loading applied to the specimens was of ca. $90 \mathrm{~N}$ with an excitation frequency of $30 \mathrm{~Hz}$. For ensuring appropriate emissivity of specimens' surfaces subjected to observation of the infrared camera, they were covered with a black matt heat-resisting silicone enamel.

During fatigue of composite structures dominated by the self-heating effect the three typical phases can be observed. In the first phase an exponential temperature increase occurs, in the second phase the self-heating temperature grows linearly, and in the third phase a rapid temperature growth appears, which finally results in a failure. The first phase can be associated with initiation of microcracks, debonding at weak fibers/matrix interface, and low strength breakage of fibers. During the second phase microcracks propagation and a macrocrack initiation can be observed. In the third phase the macrocrack propagates rapidly, and finally a failure occurs due to fiber breakage [18,19]. It should be noted that the temperature-driven fatigue occurs only when load- ing magnitudes and the resulting stresses are sufficiently high to intensify propagation of initially occurred microdamage. As it was mentioned in introduction section, if loading does not exceed a certain critical value only the two first phases occur and a value of the self-heating temperature stabilizes at a certain value. The moment of initiation of the third phase can be considered as a critical one since a rapid temperature growth can be observed. This temperature growth is related to development of a macrocrack and additional surfaces resulted from cracking, which are subjected to friction and additional heating-up of the structure.

For a purpose of determining the moment of the rapid temperature growth, which indicates initiation of the third phase of the self-heating temperature evolution, approximation of each of the self-heating temperature history curves was applied. It is assumed that the self-heating temperature can be considered as critical when a measured temperature curve starts diverging from the approximating model. For approximation, the double-exponential model was used [9]:

$T(t)=A_{1} \exp \left(B_{1} t\right)+A_{2} \exp \left(B_{2} t\right)$,

where $t$ is time; $A_{i}$ and $B_{i}$ are the pre-exponential and exponential coefficients, respectively. Parameters $A_{1}$ and $B_{1}$ are responsible for the temperature value and heating rate in the second phase of thermal fatigue, while parameters $A_{2}$ and $B_{2}$ are responsible for above-mentioned parameters in the first phase of fatigue [9]. More details on interpretation of the values of the coefficients and the reason for selection of the double-exponential model can be found in [9].

In Fig. 2, the exemplary history curve of the self-heating temperature together with its approximating model according to (1) is presented. It can be noticed that in the ca. 200th second of loading the self-heating temperature curve starts diverging from the approximating model. The reached temperature value at this moment was $80^{\circ} \mathrm{C}$. Firstly, a slight 


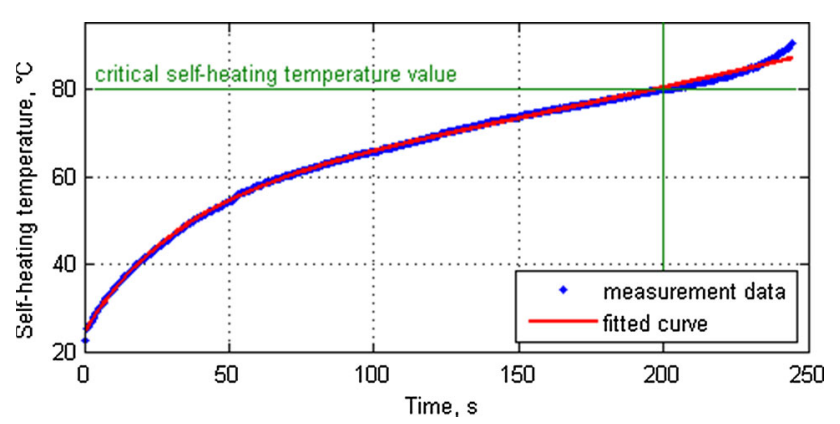

Fig. 2 Exemplary self-heating temperature curve and its approximating model

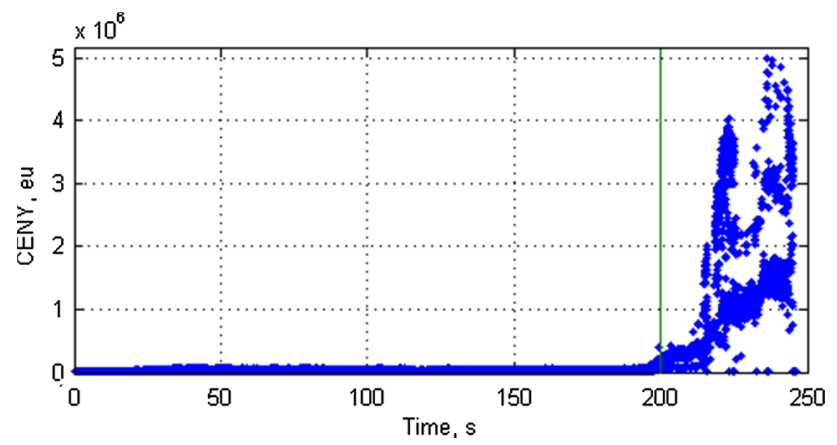

Fig. 3 Exemplary measurements of total energy of hit cascade for the specimen self-heated up to $90^{\circ} \mathrm{C}$

deviation can be observed after reaching the critical point due to the incoincidence between the experimental data and the approximation model, and then a rapid growth occurs, which is resulted by a loss in stiffness of the specimen due to a crack propagation. Analysis of all the history curves of the self-heating temperature acquired in this study indicated that the average critical value obtained based on this approach equals ca. $72^{\circ} \mathrm{C}$ (with a standard deviation equaled $8.64^{\circ} \mathrm{C}$ ).

Analysis of $\mathrm{AE}$ features was based on observation of noticeable growth of total energy of hit cascade (CENY), which is a sum of the energies of the first hit and all subse- quent hits in a hit cascade. This feature was selected since in the previous study [11] it revealed higher sensitivity to detection of the critical self-heating temperature value when compared to other AE features. Figure 3 presents the exemplary graph of CENY during loading of the same specimen as in the results presented in Fig. 2. It can be noticed that the critical moment of the self-heating based on the history curve of temperature values is very close to the moment of rapid growth of acoustic activity visible in Fig. 3 (in the about 200th second of loading). The average critical value of the self-heating based on the analysis of the AE feature of all the specimens equals ca. $69^{\circ} \mathrm{C}$ (with a standard deviation equaled $7.16^{\circ} \mathrm{C}$ ). The resulting deviations in the critical selfheating temperature values are caused by the non-stationarity of the fatigue process driven by thermal phenomena and nonstationary crack growth.

\section{Evaluation of Thermal Diffusivity}

An evaluation of TD was performed on one of the author's own-designed test rig, which is based on a heat pulse method principle (see [20] for details). A picture and a scheme of this test rig are presented in Fig. 4. A specimen holder 4 was designed to provide uniform heating of a specific region of a specimen 5 without its edges. This was ensured by applying a thermal shield, which contains a hole with smaller dimensions with respect to the tested specimen. Additionally, a thermal barrier 7 was mounted on the test rig for ensuring a thermal background for infrared (IR) measurements. As a thermal wave source a $1.2-\mathrm{kW}$ IR radiator 3 with a wavelength range of $2 \div 10 \mu \mathrm{m}$ was used, which operation temperature value of ca. $600^{\circ} \mathrm{C}$ was controlled using a temperature control unit 2 . Since the IR radiator is unable to turn on/off instantaneously, it was mounted on a moveable platform 1 , which enables moving the radiator back after the end of a heating cycle. A duration of heating was controlled



(b)

Fig. 4 A picture (a) and a scheme (b) of the own-designed test rig for thermal diffusivity measurements [20] 




Fig. 5 Exemplary dimensionless temperature history on a rear surface of a specimen obtained from the experiments

by a PLC controller 9 , which allows setting up a moment of opening of a shutter 6 (placed between a front surface of the specimen and the IR radiator) through moving by a shutter drive 8 . The thermograms were recorded with a rate of 12.5 frames per second using an IR camera ThermaCAM ${ }^{\mathrm{TM}}$ A655sc (FLIR $®$ Systems) 10 with a focal plane array detector. The data acquisition and analysis were performed using ResearchIR MAX software (FLIR® Systems). The heating duration of $1 \mathrm{~s}$ and the distance between the IR radiator and the surface of the specimen of $25 \mathrm{~mm}$ were determined experimentally, when temperature variations on the rear surface of the specimen were satisfactory for the measurements, taking into consideration the obtained temperature increase suitable for extracting the TD data. The distance from the specimen to the IR camera was approximately $0.5 \mathrm{~m}$.

The values of TD were determined using a Parker's method [13]. From the obtained plots of temperature variations versus time the dimensionless temperature history plots (see Fig. 5 for instance) were created in order to extract the $t_{0.5}$ values required for the TD calculations, according to a procedure described in [13]. The $t_{0.5}$ values, which denote a time required to reach one-half of the maximal achieved value of temperature during thermal excitation on the surface of a specimen, together with values of the specimen thickness $L$, were used to calculate the TD values according to the following equation:

$\alpha=1.38 L^{2} / \pi^{2} t_{0.5}$.

According to the above-presented method the ATD values were determined for the tested specimens for various values of the maximal self-heating temperature observed on their surfaces during fatigue tests in order to evaluate the criticality of the self-heating phenomenon.

The measurements of TD were taken according to the above-described measurement procedure. For clarity, TD

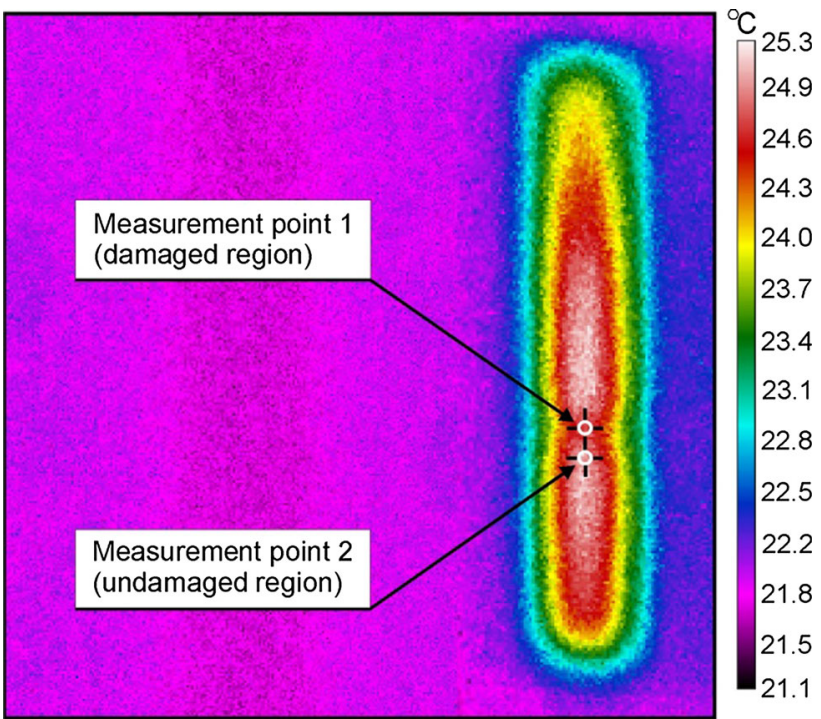

Fig. 6 Exemplary thermogram with marked measurement points

was determined in two different locations each time: Firstly, TD was determined in undamaged region of the specimen, and then, the ATD value was determined directly on the damaged region. This allows for neglecting differences resulting from measurement errors and material inhomogeneity. The average value of TD obtained from all specimens in their undamaged regions was $2.348 \times 10^{-7} \mathrm{~m}^{2} / \mathrm{s}$ (with a standard deviation of $0.015 \times 10^{-7} \mathrm{~m}^{2} / \mathrm{s}$ ). However, since small deviations from this value occur in subsequent measurements, the value of TD was determined each time during estimation of a relative ATD value on a given specimen.

\section{Analysis of the Results and Comparative Studies}

The measurements of TD and ATD were taken by acquisition of temperature history plots (see Fig. 5 for instance) extracted from points defined on each thermogram for healthy and damaged region of a specimen. The idea of these measurements is presented in Fig. 6. In this exemplary case, the thermogram (obtained near the maximum thermal contrast) of the surface of the specimen with a relatively large damaged region, one can observe a non-uniform temperature distribution along the examined part of the specimen. Such clearly visible non-uniform temperature distributions were observed only for the most damaged specimens with a difference of TD and ATD values higher than $0.06 \times 10^{-7} \mathrm{~m}^{2} / \mathrm{s}$, which corresponds to the specimens subjected to fatigue with accompanying self-heating starting from the self-heating temperature of $90^{\circ} \mathrm{C}$. The observations indicate that the thermal contrast analysis, commonly used in the non-destructive testing practice, cannot be applied for evaluation of structural degradation in case of such small damaged regions. 


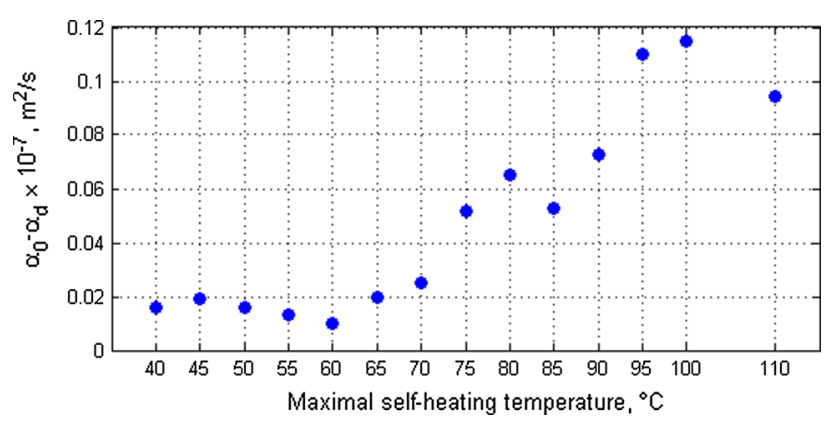

Fig. 7 Relative ATD for various self-heating temperature values

Moreover, the TD analysis-in contrast to the thermal contrast analysis-is insensitive to the non-uniform heating of a specimen surface, which is essential for quantitative interpretation of results obtained from transient thermography measurements.

Comparing the determined temperature value of $90^{\circ} \mathrm{C}$ to the results obtained using approximation of the self-heating temperature history curves and analysis of features of acoustic emission (see Sect. 2), one can conclude that the direct observation of temperature distributions for evaluation of the criticality of the self-heating effect is not appropriate.

The temperature history curves obtained from the thermographic measurements were used to determine TD and ATD values following the procedure described in Sect. 3 . The obtained values (presented in Fig. 7) are the differences between TD measured in a healthy region of a specimen $\alpha_{0}$ and ATD measured in a damaged location $\alpha_{d}$ for particular values of maximal self-heating temperature observed on the surfaces of the specimens subjected to cyclic loading.

In Fig. 7, one can observe that the value of relative ATD increases sharply for the cases where the maximal selfheating temperature observed during fatigue tests was higher than $70^{\circ} \mathrm{C}$, while for the specimens with lower self-heating temperature the relative ATD values are comparable. A small increase in the relative ATD value is observed at $70^{\circ} \mathrm{C}$, but due to a measurement error this value cannot be considered as a valid one. Following this, one can conclude that the criticality of the self-heating effect evaluated using the proposed approach based on the relative value of ATD is in the range of $70-75^{\circ} \mathrm{C}$.

Comparing the results obtained from the measurements of the relative ATD (described in Sect. 3) to the previously obtained ones from approximation of the self-heating temperature evolution curves and AE measurements (see Sect. 2), one can conclude that the approach proposed in this paper is in agreement with the previously obtained critical selfheating temperature range. The determined sensitivity of the applied method based on determination of ATD (obtained in the range of $70-75^{\circ} \mathrm{C}$ ) is comparable to the sensitivity of the other previously applied methods (approximation of the self-heating temperature curves and $\mathrm{AE}$ measurements).

\section{Conclusions}

The performed studies were focused on evaluation of structural degradation of polymer-based composites subjected to cyclic fatigue loading with accompanying self-heating effect via a novel approach based on determination of apparent thermal diffusivity in a highly stressed region of a structure. The performed experimental studies proved that the thermal diffusivity analysis is reliable for evaluation of structural degradation of polymeric composites subjected to self-heating, giving adequate quantitative results. The obtained results confirmed a critical limit of a self-heating temperature determined by other methods, which is about $70^{\circ} \mathrm{C}$ for the tested specimens. Moreover, as a method related directly to physical properties of a material, ATD-based approach reveals comparable sensitivity to the previously applied methods used for evaluation of the criticality of the self-heating effect. In contrast to the proposed ATD-based approach, the transient thermography used as a basis of determination of TD and ATD of a tested material shows low sensitivity to the occurring structural changes after loading of the tested specimens. In this case, the resulting damage after fatigue with accompanying self-heating was detectable for specimens, which reached maximal self-heating temperature of $90^{\circ} \mathrm{C}$ and higher, when the damage is well developed and visible by an unarmed eye. Therefore, the approach based on the transient thermography is not effective in evaluation of fatigue damage initiation.

The evaluated self-heating criticality can be helpful during operation of composite elements subjected to cyclic loading for preventing overloading, and the increase in a self-heating temperature up to the determined value can be considered as an indicator of fatigue damage propagation and residual life of a composite element working in such conditions.

Acknowledgements The results presented in this paper have been obtained within the framework of research Grant No. 2015/17/D/ST8/ 01294 financed by the National Science Centre, Poland.

Open Access This article is distributed under the terms of the Creative Commons Attribution 4.0 International License (http://creativecomm ons.org/licenses/by/4.0/), which permits unrestricted use, distribution, and reproduction in any medium, provided you give appropriate credit to the original author(s) and the source, provide a link to the Creative Commons license, and indicate if changes were made.

\section{References}

1. Katunin, A.; Fidali, M.: Fatigue and thermal failure of polymeric composites subjected to cyclic loading. Adv. Compos. Lett. 21(3), 64-69 (2012)

2. Rittel, D.: On the conversion of plastic work to heat during high strain rate deformation of glassy polymers. Mech. Mater. 31(2), 131-139 (1999) 
3. Lang, R.; Manson, J.A.: Crack tip heating in short-fibre composites under fatigue loading conditions. J. Mater. Sci. 22(10), 3576-3580 (1987)

4. Talreja, R.: Fatigue of Composite Materials. Technomic Publishing Company, Inc., Lancaster (1987)

5. Toubal, L.; Karama, M.; Lorrain, B.: Damage evolution and infrared thermography in woven composite laminates under fatigue loading. Int. J. Fatigue 28(12), 1867-1872 (2006)

6. Ferreira, J.A.M.; Costa, J.D.M.; Reis, P.N.B.; Richardson, M.O.W.: Analysis of fatigue and damage in glass-fibre-reinforced polypropylene composite materials. Compos. Sci. Tech. 59(10), 1461-1467 (1999)

7. Pegoretti, A.; Riccò, T.: Fatigue crack propagation in polypropylene reinforced with short glass fibres. Compos. Sci. Tech. 59(7), 1055-1062 (1999)

8. Katunin, A.; Fidali, M.: Investigation of dynamic behaviour of laminated composite plates under cyclic loading. Kompozyty 11(3), 208-213 (2011)

9. Katunin, A.: Critical self-heating temperature during fatigue of polymeric composites under cyclic loading. Compos. Theor. Pract. 12(1), 72-76 (2012)

10. Kahirdeh, A.; Khonsari, M.M.: Criticality of degradation in composite materials subjected to cyclic loading. Compos. B 61, 375-382 (2014)

11. Katunin, A.; Wronkowicz, A.; Bilewicz, M.; Wachla, D.: Criticality of self-heating in degradation processes of polymeric composites subjected to cyclic forced loading: A multiphysical approach. Arch. Civ. Mech. Eng. 17(4), 806-815 (2017)
12. Brady, R.P.; Kulkarni, M.R.: Determination of thermal diffusivity distribution for three types of materials by transient thermography. NDT\&E Int. 29(4), 205-211 (1996)

13. Parker, W.J.; Jenkins, R.J.; Butler, C.P.; Abbott, G.L.: Flash method for determining thermal diffusivity, heat capacity and thermal conductivity. J. Appl. Phys. 32(9), 1979-1984 (1961)

14. Hasselman, D.P.H.; Donaldson, K.Y.: Effects of detector nonlinearity and specimen size on the apparent thermal diffusivity of NIST 8425 graphite. Int. J. Thermophys. 11(3), 573-585 (1990)

15. dos Santos, W.N.; Mummery, P.; Wallwork, A.: Thermal diffusivity of polymers by the laser flash technique. Polym. Test. 24(5), 628634 (2005)

16. Navarrete, M.; Serranía, F.; Villagrán, M.: Application of the flash method for the thermal characterisation of woven carbon fibre laminates. Mater. Des. 22(2), 93-97 (2001)

17. Katunin, A.: Thermal fatigue of polymeric composites under repeated loading. J. Reinf. Plast. Compos. 31(15), 1037-1044 (2012)

18. Naderi, M.; Khonsari, M.M.: A thermodynamic approach to fatigue damage accumulation under variable loading. Mater. Sci. Eng. A 527, 6133-6139 (2010)

19. Naderi, M.; Kahirdeh, A.; Khonsari, M.M.: Dissipated thermal energy and damage evolution of Glass/Epoxy using infrared thermography and acoustic emission. Compos. B 43(3), 1613-1620 (2012)

20. Pawlak, S.: Thermographic evaluation method of the fiber content distribution in Carbon-Epoxy composites, PhD Thesis, Silesian University of Technology, Gliwice (2010). (in Polish) 